4-1-2021

\title{
Karfafa amfani da kwayoyin hana daukar ciki na zamani a tsakanin mata a jihar Zamfara
}

Breakthrough RESEARCH

Follow this and additional works at: https://knowledgecommons.popcouncil.org/departments_sbsr-rh How does access to this work benefit you? Let us know!

\section{Recommended Citation}

Breakthrough RESEARCH. 2021. "Karfafa amfani da kwayoyin hana daukar ciki na zamani a tsakanin mata a jihar Zamfara," infographic. Washington, DC: Population Council. 


\section{Karfafa amfani da kwayoyin hana daukar ciki na zamani a tsakanin mata a jihar Zamfara}

Aikin Breakthrough RESEARCH ya gudanar da bincike a watan Satumba 2019 a kan dabi'un mata game da kula da lafiya a lokacin da suka sami juna-biyu ko suka haihu a cikin shakaru biyu da suka wuce.

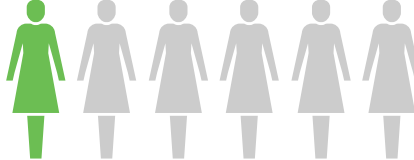

Mace daya (1) ne kowai cikin mata shida (6) $(15 \%)$ a cikin biyar (5) daga cikin kananan hukomin Jihar Zamfara da aka gudanar da bincike ke amfani da kwayoyin hana daukar ciki na zamani.

\section{Sakamakon Nazarin Jihar Zamfara}

Nazarin lura da halayyar ya gano muhimman abubuwa guda uku don inganta amfani da kwayoyin hana daukar ciki na zamani. Kashin da aka nuna a kasa sune kasa da tsaka-tsakin makin na duk kananan hukumomin da aka zaba domin binciken. Matsakaitan adadin za su iya shan bambam da matsaikacin maki da aka nuna a shafin na gaba.

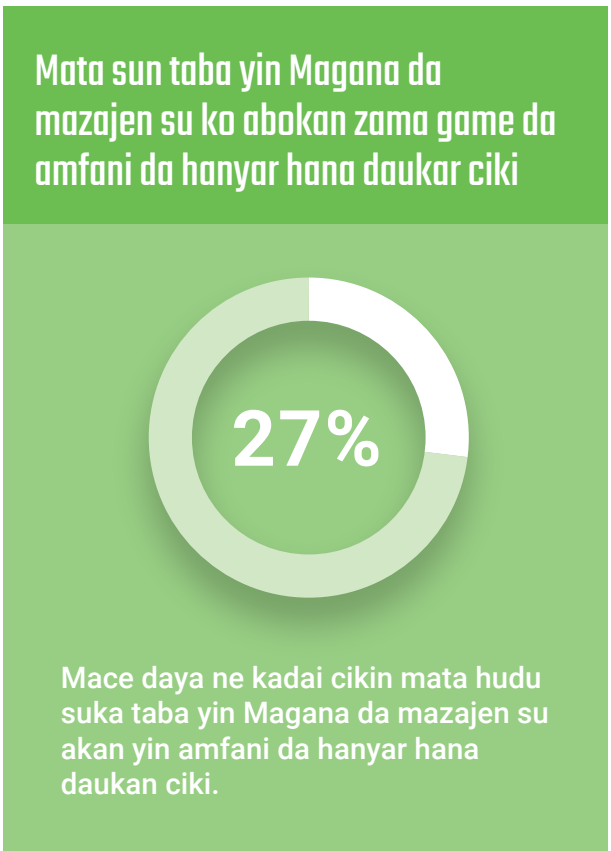

\section{Mata da kansu sun yarda da yin amfani da kwayoyin hana daukar cikin domin tazarar haihuwa}

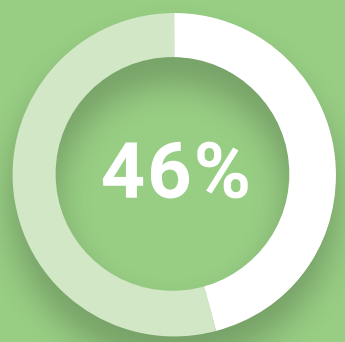

Kasa da rabin mata sun yarda da kan su akan yin amfani da kwaoyin hana daukar ciki domin tsara iyali.
Mata suna da ilimi mai yawa akan tsara iyali ${ }^{\dagger}$

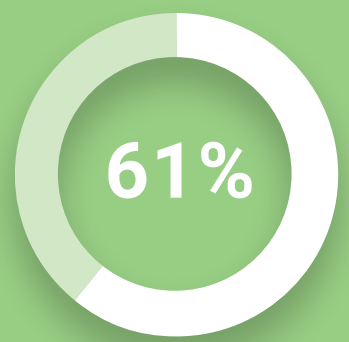

Kimanin mata shida cikin goma suna da ilimi sosai akan tsara iyali.

${ }^{\dagger}$ An yi la'akari da sanin amfanin tsara iyali game da mata da yaran su, da sanin cewa yin amfani da kywayoyin hana daukar ciki na da wasu illoli sanannue, da amfanin haihuwan yara bayan shekaru 18 da kuma illolin kunika wajen auna yawan ilimi akan tsara iyali.

\section{Idan an kara inganta waddanan abubuwan, neman magani zai iya karuwa a jihar Zamfara.}

\section{Sakamakon Nazari Na Kananan Hukumomi a Jihar Zamfara}

An taba yin Magana da maigida akan amfan da hanyar hana daukar ciki Namoda

Bungudu

Talata Mafara

Tsafe

Gusau

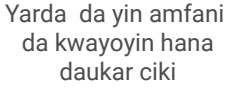

Yarda da yin amfani da kwayoyin hana daukar ciki
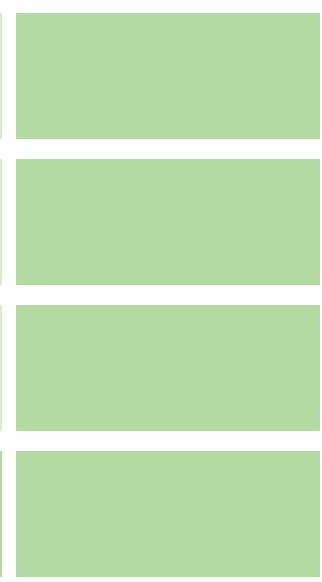

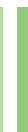

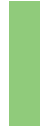

$\mathrm{Na}$ da ilimi sosai akan tsara iyali
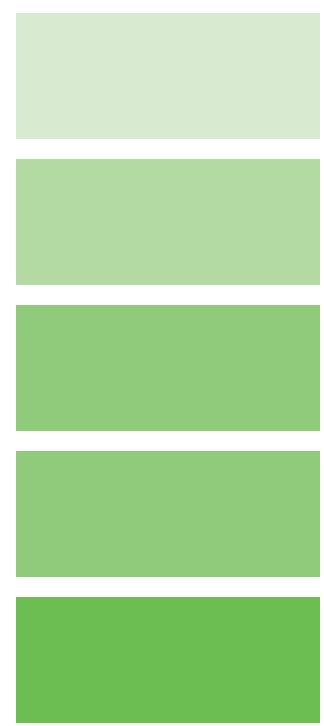
Na amfani da kwayoyin
hana daukar ciki na zamani

Kaso na mata wandanda suka bada tabbataccen amsa da cikin kanan hukumomi

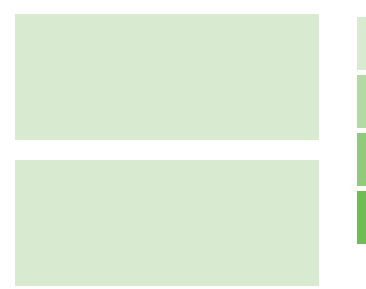

$$
\begin{aligned}
& 0 \%-24 \% \\
& 25 \%-49 \% \\
& 50 \%-74 \% \\
& 75-100 \%
\end{aligned}
$$




\section{Karfafa amfani da kwayoyin hana daukar ciki na zamani a tsakanin mata a jihar Zamfara}

Aikin Breakthrough RESEARCH ya gudanar da bincike a watan Satumba 2019 a kan dabi'un mata game da kula da lafiya a lokacin da suka sami juna-biyu ko suka haihu a cikin shakaru biyu da suka wuce.

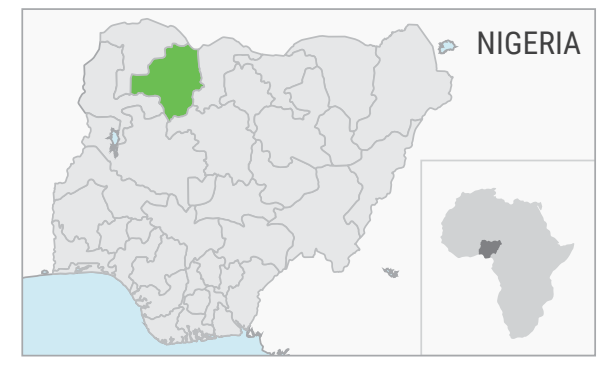

\section{Sakamakon binciken da aka yi ta jihar Zamfara}

Binciken kan dabi'un matan ya gano cewa, muhimman abubuwa guda uku ne da zai inganta yin amfani da kwayoyin hana daukar ciki na zamani. Adadin kowanne maki a cikin akwati shi ne maki kasa da tsaka-tsakin makin (kashi 50 daga cikin dari). Rabin kananan kukumomi sun samu makin fiye da hamsin (masu launin ja) kuma sauran kananan hukumomi sun samu maki fiye da hamsin (masu launin kore).

\section{Mata sun taba yin Magana da mazajen su ko abokan zama game da amfani da hanyar hana daukar ciki}

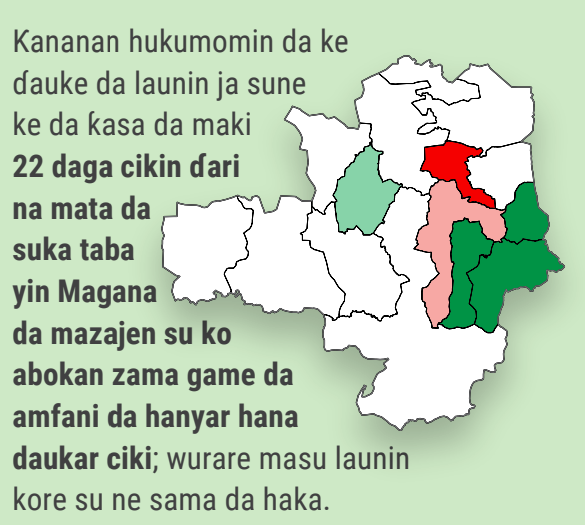

\section{Mata da kansu sun yarda da yin amfani da kwayoyin hana daukar cikin domin tazarar haihuwa}

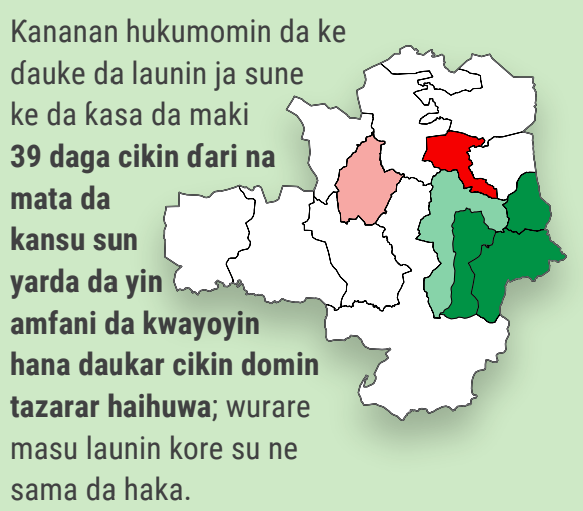

\section{Mata suna da ilimi mai yauwa akan} tsara iyali

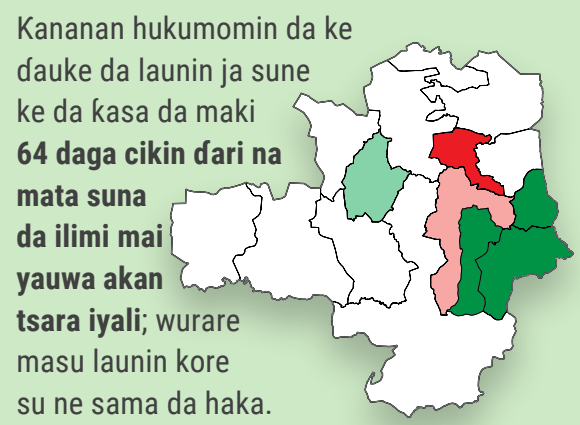

Karin bayani: Bangaren da aka sanya wa launi mai haske sune ke dab da mataki na kasan ko saman tsakiya. Yankuna masu launi duhu-duhu, sun nesanta da na tsakiya, a inda yake nuni da kasa da kashi 25 daga cikin dari ko sama da kashi 75 daga cikin dari. Kananan hukumomi da ke dauke da launin fari ba sa cikin samfurin nazarin. Saboda haka, babu bayanin maki kamar yadda aka yi wa sauran.

\section{Ta ya ya ne za a yi amfani da wannan bayani don inganta yin amfani da kwayoyin hana daukar ciki na zamani a jihar Zamfara?}

Tattaunawa da tambayoyi akan kalubalen da aka fuskanta aabubuwan da suke taimakawa wajen yin amfani da kwayoyin hana daukar ciki na zamani na iya samun mahanga akan yanda za a inganta taimako a waddanan bangarori ta hanyar yin tambayoyi kamar haka:

- Mene ne ya bambanta a shiyoyin kananan hukumomin da ke dauke da launin kore da zai iya bayyana nagartar tafyar da aikinsu a kananan asibitocin? Shin, ko kananan hukumomi masu dauke da launin kore suna samu karin tallafin kayan aiki daga waje da kuma samun horon inganta kwarewa wajen tafiyar da aikinsu da na wayar da kan al'umma?

- Mene ne zai bambanta al'ummomin da zasu iya bayar da cikakken bayanin irin gudumawar da kananan hukumomi ke bayar wa a fagen lura da lafiya? Shin, ko suna da jaruman shugabanni da nagartattun kwamitoci a kowanne yankin, ko ana bayar da dama ga 'yan yankin da su shigo domin bayar da gudunmawa a kan harkar bunkasa dorewar kula da lafiyar jama'ar yankin?

- Wasu kalubale ne a cikin kananan hukumomi masu launin ja da suke tasiri akan muhiman abubuwa uku da aka lissafa wato ilimin tsara iyali, yardan mata da kuma yin Magana da mazajen su , da kuma yanda abubuwan nan suke hana mata yin amfani da kwayoyin hana daukar ciki na zamani? Ta ya ya ne shugabanni da sauran al'ummar yankin za su iya kawo karshen wadannan kalubalen?

- Shin kananan hukumomin masu launin kore na da abubuwan da ke kara ilimin mata, da yarda, da daman yin Magana da mazajen su da kuma yin amfani da kwayoyin hana daukar ciki na zamani? Ta ya ya za su yi amfani da hakan a dace a yankuna masu karancin amfani da kwayoyin hana daukar ciki na zamani?

- Ta yay a za a inganta aikin ko bayanai zuwa ga al'ummomi da gidajen da ke cikin matukar bukatar ingantacen bayanai a kan muhimancin amfani da kwayoyin hana daukar ciki?

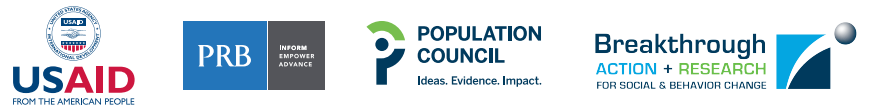

Wannan Bincike mai lakanin Breakthrough RESEARCH ya samu tabbata ne tare da kyakkyawar tallafin mutanen amurka ta hanun hukumar kula da cigaban kasashe na amurka (USAID) a karkashin yarjejeniya ta hadin kai mai lamba AID-OAA-A-17-00018. Bayanan da wannan kundi ya kunsa ra'ayi ne na wannan binciken Breakthrough RESEARCH da kuma hukumar Population Council, bai zama tilas yana da alaka da ra'ayin hukumar USAID ba ko na gwamnatin Amurka. 